\title{
A Metastatic Ovarian Angiosarcoma Mimicking Hematologic Neoplasia at Diagnosis
}

\author{
Rafael Dezen Gaiolla, b Ívison Xavier Duarte ${ }^{c}$ Carlos Eduardo Bacchi ${ }^{c}$ \\ Carlos Eduardo Paiva ${ }^{d}$ \\ ${ }^{a}$ Hematology Service, Department of Internal Medicine, Botucatu Medical School, São \\ Paulo State University (UNESP), ${ }^{\mathrm{b}}$ Institute of Oncology and Hematology - ONCOMED, and \\ ${ }^{c}$ Consultoria em Patologia, Botucatu, and ${ }^{d}$ Department of Clinical Oncology, Barretos \\ Cancer Hospital, Barretos, Brazil
}

\section{Key Words}

Angiosarcoma $\cdot$ Bone marrow metastasis $\cdot$ Splenomegaly $\cdot$ Bone marrow cytology

\begin{abstract}
Angiosarcomas are rare aggressive neoplasms of vascular endothelial origin with a high metastatic rate and poor prognosis. Involvement of the bone marrow by the angiosarcoma is exceedingly uncommon, and there have only been a few cases reported in the literature to date. Clinical manifestations and common laboratory findings of bone marrow involvement can mimic other more common bone marrow-replacing neoplasias such as lymphomas and acute leukemia. A definitive diagnosis is difficult to make from cytologic material, probably due to an associated bone marrow fibrosis, and requires bone marrow trephine biopsy with an immunohistochemical profile. Here we had the opportunity to study a case of metastatic angiosarcoma with positive cytologic findings and an unusual presentation that challenged its primary diagnosis.

(c) 2014 S. Karger AG, Basel
\end{abstract}

\section{Background}

Angiosarcomas are rare aggressive neoplasms of vascular endothelial origin with a high metastatic rate, accounting for almost $4 \%$ of all soft tissue sarcomas [1]. The median age at diagnosis is 50-60 years and the most common sites of presentation are the skin (mainly in the head and neck area) and soft tissues [2], but primary involvement of the breast, liver, spleen and bone, although rare, has also been described [1]. Involvement of the bone

Rafael Dezen Gaiolla

Botucatu Medical School, Department of Internal Medicine

Distrito de Rubião Junior

Botucatu, SP 18618-970 (Brazil)

E-Mail rgaiolla@fmb.unesp.br 
marrow by the angiosarcoma is exceedingly uncommon and almost invariably found in patients with disseminated disease, usually with concomitant involvement of the spleen. To our knowledge, there have only been a few cases with bone marrow infiltration reported in the literature to date [3-6].

Clinical manifestations of bone marrow involvement by the angiosarcoma are nonspecific and include fatigue, weight loss, mucocutaneous bleeding and fever. Common laboratory findings are anemia and thrombocytopenia, which can be found in many patients. A leukoerythroblastic blood picture is also commonly observed [7]. A definitive diagnosis is difficult to make from cytologic material because of the technical difficulty in marrow aspiration and the paucity of diagnostic cells, probably due to an associated bone marrow fibrosis [8]. Therefore, the diagnosis relies on bone marrow trephine biopsy findings confirmed by the immunohistochemical profile. In general, the prognosis of metastatic angiosarcoma is very poor, with a median overall survival of less than 12 months $[9,10]$. The mainstay treatment of metastatic angiosarcoma is not well defined. There are no randomized trials, and most published reports are based on retrospective case series $[9,10]$ with response rates varying between 16 and 36\% [11]. Systemic single-agent chemotherapy has been the most common treatment approach, although the evidence base for this approach is limited. Anthracycline-based regimens and weekly paclitaxel remain the most commonly used first-line treatments in the metastatic setting [12]. Weekly paclitaxel has been shown to be an active agent in metastatic angiosarcomas [11], and its benefit appears to be more pronounced in primary angiosarcomas arising in the scalp or breast [2]. Here, we describe the case of an angiosarcoma metastatic to the spleen and bone marrow, an extremely unusual presentation that challenges the primary diagnosis. Written informed consent was obtained from the patient's daughter for publication of this case report and any accompanying images.

\section{Case Presentation}

A 71-year-old woman was referred to the Hematology Clinic after a routine evaluation in which a complete blood count revealed anemia and thrombocytopenia. The patient noticed a cumulative weight loss of $12 \mathrm{~kg}$ in the previous year and progressive fatigue during the last 2 months. She denied fever, dyspnea, repeated infections or abdominal pain. There was a history of a gastric neuroendocrine tumor 2 years ago, which was surgically removed. The patient admitted that she had not had a gynecological follow-up for years. A physical examination revealed pallor, an exclusive left lobe hepatomegaly and massive splenomegaly with no palpable lymph node. The breast examination was normal. She had a poor performance status of 2 . The peripheral blood counts revealed a hemoglobin level of $74 \mathrm{~g} / \mathrm{l}$ (mean corpuscular volume $95 \mathrm{fl}$ ), platelet levels of $53 \times 10^{9} / \mathrm{l}$ and a white blood cell count of $7.7 \times$ $10^{9} / \mathrm{l}$ with a 4.9 absolute neutrophil count.

A bone marrow aspiration was dry tap. An imprint from the bone marrow biopsy was performed and showed moderate megaloblastic changes with a few groups of cohesive nonhematopoietic mononuclear cells with round to indented nuclei, homogeneously condensed chromatin, distinct nucleoli and deeply basophilic cytoplasm (fig. 1). The bone marrow trephine biopsy revealed replacement of the normal hematopoietic elements by interstitial infiltration of malignant epithelioid cells with abundant eosinophilic cytoplasm, focal spindle-shaped cells lining cleft-like spaces and anastomosing vascular channels (fig. 2). An immunohistochemical analysis revealed that tumor cells expressed the endothelial markers CD31, factor VIII (fig. 3) and CD34 with no expression of epithelial markers, such as 
Gaiolla et al:: A Metastatic Ovarian Angiosarcoma Mimicking Hematologic Neoplasia at Diagnosis

cytokeratin. HHV-8 was negative. The morphological and immunohistochemical findings were indicative of bone marrow infiltration by angiosarcoma.

The patient was referred to the Oncology Clinic for evaluation. Further workup was performed to identify other sites of involvement. Electrolytes, creatinine, lactate dehydrogenase and liver tests were within normal limits. The cancer antigen (CA) 15.3 level was slightly elevated $(30 \mu \mathrm{g} / \mathrm{l}$, normal $<25 \mu \mathrm{g} / \mathrm{l})$. The carcinoembryonic antigen, CA 19.9 and CA 125 levels were in the normal range. The patient underwent CT scans of the chest, abdomen and pelvis, which showed homogeneous enlargement of the liver and spleen. Bone scintigraphy was normal. A transvaginal ultrasound revealed an enlarged right ovary measuring $4.4 \mathrm{~cm}$ in the greatest diameter with a multisepted lesion. Upper gastrointestinal endoscopy and colonoscopy were performed, both with normal findings. With these results, we presumed the presence of a primary ovarian angiosarcoma with simultaneous involvement of the spleen, bone marrow and liver. Although the ovarian tumor was not biopsied, its radiological appearance together with the assumption that metastatic sarcoma is very rare in the ovary directed us to assume that the ovarian tumor was the primary site. In addition, no other plausible primary tumor was found in the workup. Systemic chemotherapy was recommended, and the patient began monotherapy with paclitaxel $\left(80 \mathrm{mg} / \mathrm{m}^{2}\right.$, intravenously, weekly on days $1,8,15$ every 28 days). After 2 cycles, the patient developed a grade 3 peripheral neuropathy, and the treatment was halted. At that time, new CT scans showed a stable disease with a slight improvement in hemoglobin levels. The treatment was modified, and the patient underwent chemotherapy with pegylated liposomal doxorubicin $\left(35 \mathrm{mg} / \mathrm{m}^{2}\right.$, on day 1, intravenously, every 3 weeks). At the end of 6 cycles, there was a strong improvement in the hemoglobin levels to $119 \mathrm{~g} / \mathrm{l}$ and a normalization of the platelet counts. A PETCT was performed and revealed a complete reduction of the splenomegaly but no improvement in the hepatomegaly. In addition, a highly active metabolic lesion was still present in the right ovary. The bone marrow biopsy was repeated and showed a persistence of angiosarcoma cells. The patient showed an asymptomatic partial response with moderate treatment toxicity; therefore, it was decided to maintain the clinical follow-up until symptomatic progression. Seven months later, the patient started experiencing bone pain, and a new bone scintigraphy revealed diffuse osteoblastic lesions. The chest and abdominal CTs were repeated showing a $0.8-\mathrm{cm}$ lung nodule as well as multiple liver metastases. Chemotherapy with gemcitabine $\left(1,000 \mathrm{mg} / \mathrm{m}^{2}\right.$, intravenously, on days 1,8 and 15 every 28 days) plus zoledronic acid was initiated with no improvement after 2 cycles, and there was a significant decrease in the performance status. The patient died 5 months after palliative care initiation, 22 months after the diagnosis had been established.

\section{Conclusions}

Angiosarcomas can metastasize to the bone marrow, leading to a clinical picture that eventually mimics other more common bone marrow-replacing diseases, such as acute leukemia and non-Hodgkin lymphoma. In this sense, a high index of suspicion is necessary due to its rarity. Oncologists and hematologists need to be aware of this challenging diagnosis and to consider bone marrow evaluation, including cytology smear, in patients with angiosarcoma and peripheral blood count abnormalities. 
Gaiolla et al.: A Metastatic Ovarian Angiosarcoma Mimicking Hematologic Neoplasia at Diagnosis

\section{Acknowledgements}

The authors would like to thank the staff of Consultoria em Patologia for their technical assistance in histological and immunohistochemical techniques.

\section{Disclosure Statement}

The authors declare that they have no conflict of interests.

\section{References}

1 Lewis CJ, Gerrand C, Barnes DE, Murray S, Milner RH, Ragbir M: Experience of angiosarcoma in the North of England Bone and Soft Tissue Tumour Service. J Plast Reconstr Aesthet Surg 2011;64:884-891.

-2 Penel N, Marreaud S, Robin YM, Hohenberger P: Angiosarcoma: state of the art and perspectives. Crit Rev Oncol Hematol 2011;80:257-263.

-3 So CC, Wong KF: Bone marrow involvement by angiosarcoma. Br J Haematol 2001;115:1.

4 Smith RA, Dzugan SA, Rafique S, Lucas M: Angiosarcoma replacing bone marrow - A case report. J Miss State Med Assoc 2005;46:35-36.

5 Wang C, Rabah R, Blackstein M, Riddell RH: Bone marrow metastasis of angiosarcoma. Pathol Res Pract 2004;200:551-555.

6 Noack F, Balleisen L, Valent P, Horny HP: Angiosarcoma of bone marrow with unusual expression of chymase: diagnosis in a trephine biopsy specimen. J Clin Pathol 2007;60:1183.

7 Hsu JT, Chen HM, Lin CY, Yeh CN, Hwang TL, Jan YY, Chen MF: Primary angiosarcoma of the spleen. J Surg Oncol 2005;92:312-316.

-8 Wong KF, So CC, Wong N, Siu LL, Kwong YL, Chan JK: Sinonasal angiosarcoma with marrow involvement at presentation mimicking malignant lymphoma: cytogenetic analysis using multiple techniques. Cancer Genet Cytogenet 2001;129:64-68.

-9 Abraham JA, Hornicek FJ, Kaufman AM, Harmon DC, Springfield DS, Raskin KA, Mankin HJ, Kirsch DG, Rosenberg AE, Nielsen GP, et al: Treatment and outcome of 82 patients with angiosarcoma. Ann Surg Oncol 2007;14:1953-1967.

10 Fayette J, Martin E, Piperno-Neumann S, Le Cesne A, Robert C, Bonvalot S, Ranchere D, Pouillart P, Coindre JM, Blay JY: Angiosarcomas, a heterogeneous group of sarcomas with specific behavior depending on primary site: a retrospective study of 161 cases. Ann Oncol 2007;18:2030-2036.

11 Young RJ, Brown NJ, Reed MW, Hughes D, Woll PJ: Angiosarcoma. Lancet Oncol 2010;11:983-991.

12 Serrano C, Garcia A, Brana I, Perez-Benavente A, Oaknin A: Angiosarcoma of the ovary: is it always a lethal disease? J Clin Oncol 2010;28:e675-e677. 


\section{Case Reports in Oncology}

\begin{tabular}{l|l}
\hline Case Rep Oncol 2014;7:260-265 \\
\hline DOI: $10.1159 / 000362489$ & $\begin{array}{l}\text { C 2014 S. Karger AG, Basel } \\
\text { www.karger.com/cro }\end{array}$ \\
\hline
\end{tabular}

Gaiolla et al.: A Metastatic Ovarian Angiosarcoma Mimicking Hematologic Neoplasia at Diagnosis

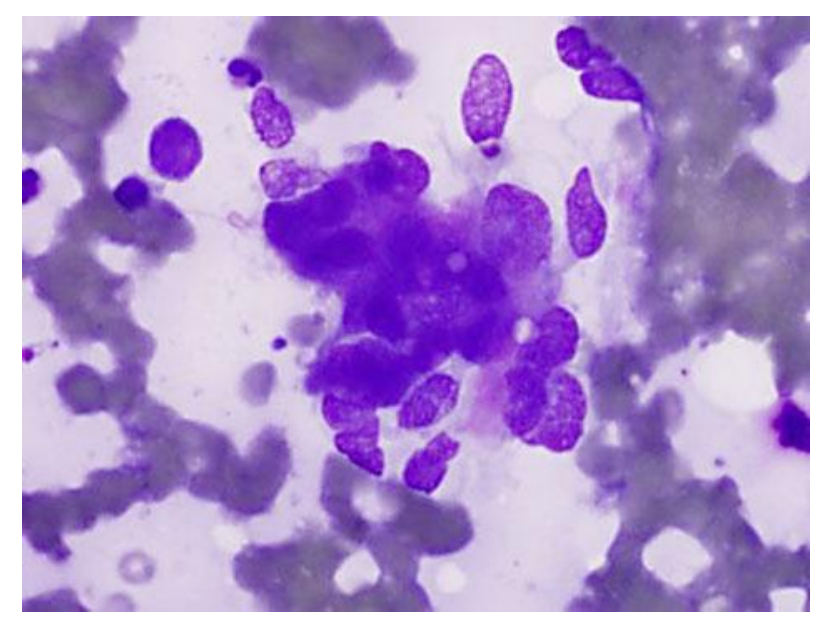

Fig. 1. Imprint from bone marrow biopsy. Giemsa stain. $\times 1,000$.

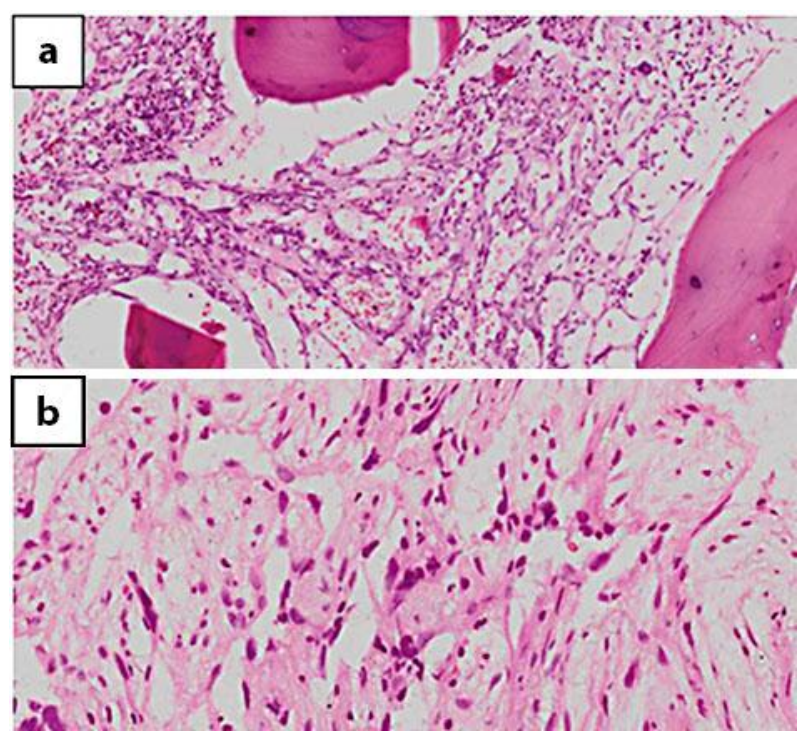

Fig. 2. Bone marrow trephine biopsy. a Intertrabecular space completely filled by angiosarcoma. The lesion shows multiple anastomosing vascular channels (HE. $\times 100)$. b Vascular spaces lined by endothelial cells with mild to moderate atypia, surrounded by spindled neoplastic cells (HE. $\times 400)$. 
Gaiolla et al.: A Metastatic Ovarian Angiosarcoma Mimicking Hematologic Neoplasia at Diagnosis

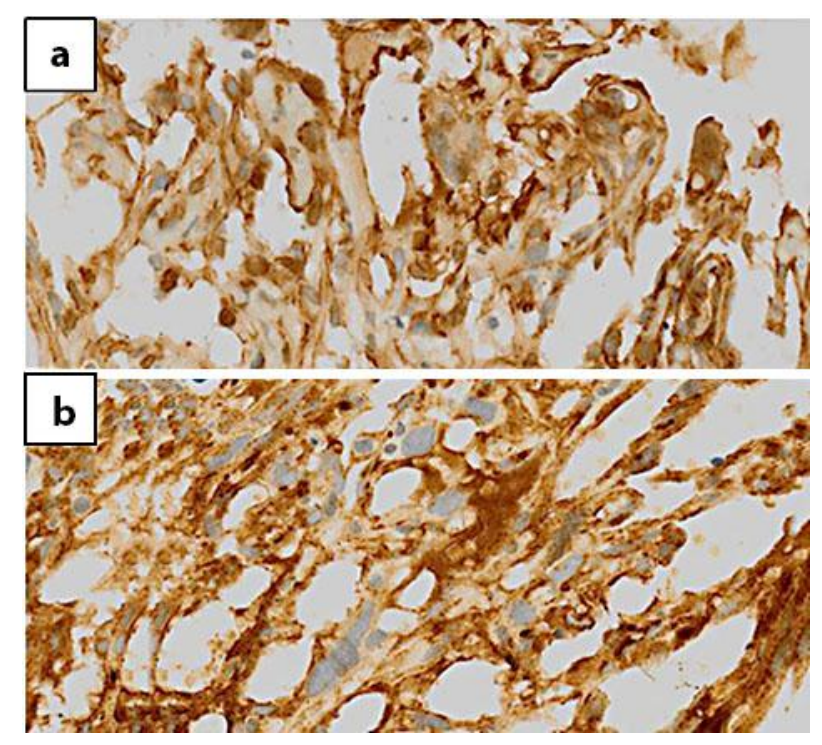

Fig. 3. Immunohistochemical staining of the bone marrow biopsy. a CD31 positivity in neoplastic cells $(\times 400)$. b Factor VIII positivity in neoplastic cells $(\times 400)$. 